\title{
Improved genome annotation through untargeted detection of pathway-specific metabolites
}

\author{
Benjamin P Bowen ${ }^{1 *}$, Curt R Fischer ${ }^{2}$, Richard Baran ${ }^{1}$, Jillian F Banfield ${ }^{2,3}$, Trent Northen ${ }^{1}$
}

\begin{abstract}
Background: Mass spectrometry-based metabolomics analyses have the potential to complement sequence-based methods of genome annotation, but only if raw mass spectral data can be linked to specific metabolic pathways. In untargeted metabolomics, the measured mass of a detected compound is used to define the location of the compound in chemical space, but uncertainties in mass measurements lead to "degeneracies" in chemical space since multiple chemical formulae correspond to the same measured mass. We compare two methods to eliminate these degeneracies. One method relies on natural isotopic abundances, and the other relies on the use of stableisotope labeling (SIL) to directly determine $C$ and $N$ atom counts. Both depend on combinatorial explorations of the "chemical space" comprised of all possible chemical formulae comprised of biologically relevant chemical elements.

Results: Of 1532 metabolic pathways curated in the MetaCyc database, 412 contain a metabolite having a chemical formula unique to that metabolic pathway. Thus, chemical formulae alone can suffice to infer the presence of some metabolic pathways. Of 248,928 unique chemical formulae selected from the PubChem database, more than $95 \%$ had at least one degeneracy on the basis of accurate mass information alone. Consideration of natural isotopic abundance reduced degeneracy to 64\%, but mainly for formulae less than $500 \mathrm{Da}$ in molecular weight, and only if the error in the relative isotopic peak intensity was less than $10 \%$. Knowledge of exact $\mathrm{C}$ and $\mathrm{N}$ atom counts as determined by SIL enabled reduced degeneracy, allowing for determination of unique chemical formula for $55 \%$ of the PubChem formulae.

Conclusions: To facilitate the assignment of chemical formulae to unknown mass-spectral features, profiling can be performed on cultures uniformly labeled with stable isotopes of nitrogen $\left({ }^{15} \mathrm{~N}\right)$ or carbon $\left({ }^{13} \mathrm{C}\right)$. This makes it possible to accurately count the number of carbon and nitrogen atoms in each molecule, providing a robust means for reducing the degeneracy of chemical space and thus obtaining unique chemical formulae for features measured in untargeted metabolomics having a mass greater than $500 \mathrm{Da}$, with relative errors in measured isotopic peak intensity greater than 10\%, and without the use of a chemical formula generator dependent on heuristic filtering. These chemical formulae can serve as indicators for the presence of particular metabolic pathways.
\end{abstract}

\section{Background}

Untargeted profiling of small molecule metabolites using mass spectrometry has the potential to aid in the functional annotation of genomes. Comprehensive metabolite identification in untargeted metabolomics experiments would greatly improve downstream analyses, including

\footnotetext{
* Correspondence: BPBowen@lbl.gov

'Department of GTL Bioenergy and Structural Biology, Life Sciences Division, Lawrence Berkeley National Laboratory, 1 Cyclotron Road, Berkeley, CA 94720, USA

Full list of author information is available at the end of the article
}

metabolic network reconstruction [1,2] and metabolomics-aided genome annotation [3,4]. Specifically, detection of a compendium of metabolites in given organisms or communities can improve confidence in pathway-extension or hole-filing for sparsely annotated pathways [5-7]. In this manner, metabolomics provides an orthogonal resource that can complement sequence homology-based methods of genome annotation.

Identification of metabolites in untargeted mass spectrometry-based metabolomics using retention time, mass, and fragmentation pattern information remains a
C Biomed Central

() 2011 Bowen et al; licensee BioMed Central Ltd. This is an open access article distributed under the terms of the Creative Commons Attribution License (http://creativecommons.org/licenses/by/2.0), which permits unrestricted use, distribution, and reproduction in any medium, provided the original work is properly cited. 
challenge [8], and validation of possible identifications by comparison to commercially available chemical standards is only possible for a subset of cases [9]. De novo identification of metabolites from spectral features or fragmentation (MS/MS) spectra is a tedious process and is currently not reliably scalable to large experiments [10]. However, the identification of a metabolite's chemical formula is a more tractable challenge, and formula assignment provides partial information about the identity of the observed metabolite. Typically, mass alone is not sufficient to specify the chemical formula $[11,12]$.

The most common approach begins with combinatorial generation of possible chemical formulae that might correspond to a detected mass spectral feature. The astronomical number of possible formulae means that heuristic limitations are required to guide this combinatorial search. The most common restriction is to limit the elements that might comprise a detected ion to only those that are most biologically relevant: carbon, hydrogen, nitrogen, oxygen, sulfur, and phosphorus. Thus, formula generators must explore all possible formulae of the form $\mathrm{C}_{a} \mathrm{H}_{b} \mathrm{~N}_{c} \mathrm{O}_{x} \mathrm{~S}_{y} \mathrm{P}_{z}$, which spans a six dimensional space, where the dimensions are $a, b, c, x, y$, and $z$. For small molecule metabolites, maximal values for these dimensions might be close to 200 carbons and hydrogens, and lesser numbers of heteroatoms (see Materials and Methods), which still allows for a search space of $288,120,000$ possible formulae.

Further heuristic restrictions, for example based valence requirements, have been used in some formula generating algorithms $[11,12]$. Relative isotope abundance patterns are reproducible and can be used to constrain likely chemical formula [13-15]. However, even when using restricted chemical formulae and isotopic data, the degeneracy around a mass value can still be high. A conceptual way to understand this point is to view mass as a single-dimensional projection of the six dimensional chemical space. Other information embedded in mass spectral data can serve as non-massbased criteria to restrict the range of possible chemical formulae. The development of certain heuristics for prioritizing the likelihood of chemical formulae reduces the number of possible chemical formulae, but leaves some ambiguity that can be reduced through additional experimentation $[11,12]$.

Modern mass spectrometers can constrain compound masses to within a few parts per million (ppm). Such accurate measurements assist in the task of determining chemical formulae (e.g., time-of-flight, ion-trap, and ion cyclotron resonance (ICR) mass spectrometers), especially when the mass of the target compound is large. Fourier transform ICR (FT-ICR) mass spectrometers have sufficient mass resolution and accuracy to enable use of isotopic fine structure for direct formula assignment. However, the majority of instruments used for untargeted metabolomics do not have such high resolution. In addition, to accurate mass measurements, accurate measurements of isotopic peak intensities are critical if natural isotopic abundance information is to be used. The importance of accurate intensity information increases as the mass of the target compound increases.

Notably, the use of stable isotope labeling has been shown to reduce the ambiguity of chemical formula assignment and has tremendous potential to aid in the comprehensive profiling of small molecules to better understand physiology [16-19]. Stable isotope labeling methods allow counting of $\mathrm{C}$ and $\mathrm{N}$ per formula unit and can lead to identification of the chemical formula without reliance on the natural isotopic abundance patterns and without using a restricted chemical formula generator.

In the current study, we compare chemical formula identification using natural isotopic abundance patterns to stable isotope labeling methods. We compare direct measurement of the counts of carbon and nitrogen atoms in an empirical formula to natural isotopic abundance information as a way to restrict chemical formula assignment. In addition, we show that simply identifying chemical formulae is sufficient to infer biological pathways. Thus untargeted metabolomics studies can inform genome annotation.

\section{Results and discussion}

\section{Linking empirical formulas to metabolic pathways}

To test the hypothesis that empirical formulae alone could pinpoint the presence of a particular metabolic pathway, we examined the MetaCyc collection of pathways and metabolites [20]. This analysis showed that many unique (not present in any other pathway or reaction not related to a pathway) metabolites and compounds with unique chemical formulae participate in a small number of reactions or pathways (Figure 1). Taking into account metabolites consisting of $\mathrm{C}, \mathrm{H}, \mathrm{N}, \mathrm{O}, \mathrm{S}$, and $\mathrm{P}$, there are 1532 pathways in MetaCyc that are not "Super-Pathways" (aggregates of multiple pathways). Of these pathways, 721 have at least one unique metabolite (Additional File 1). Additionally, 412 of these pathways have at least one metabolite with a unique chemical formula (Additional File 1). Identification of specific metabolites or merely the chemical formulae of detected metabolites may thus indicate the presence of specific reactions or pathways. This evidence cannot be considered conclusive, as specific metabolites can participate in reactions or pathways not covered by MetaCyc. Also, specific chemical formulae may correspond to metabolites not covered by MetaCyc or any known biological database. That said, mapping sets of experimentally 


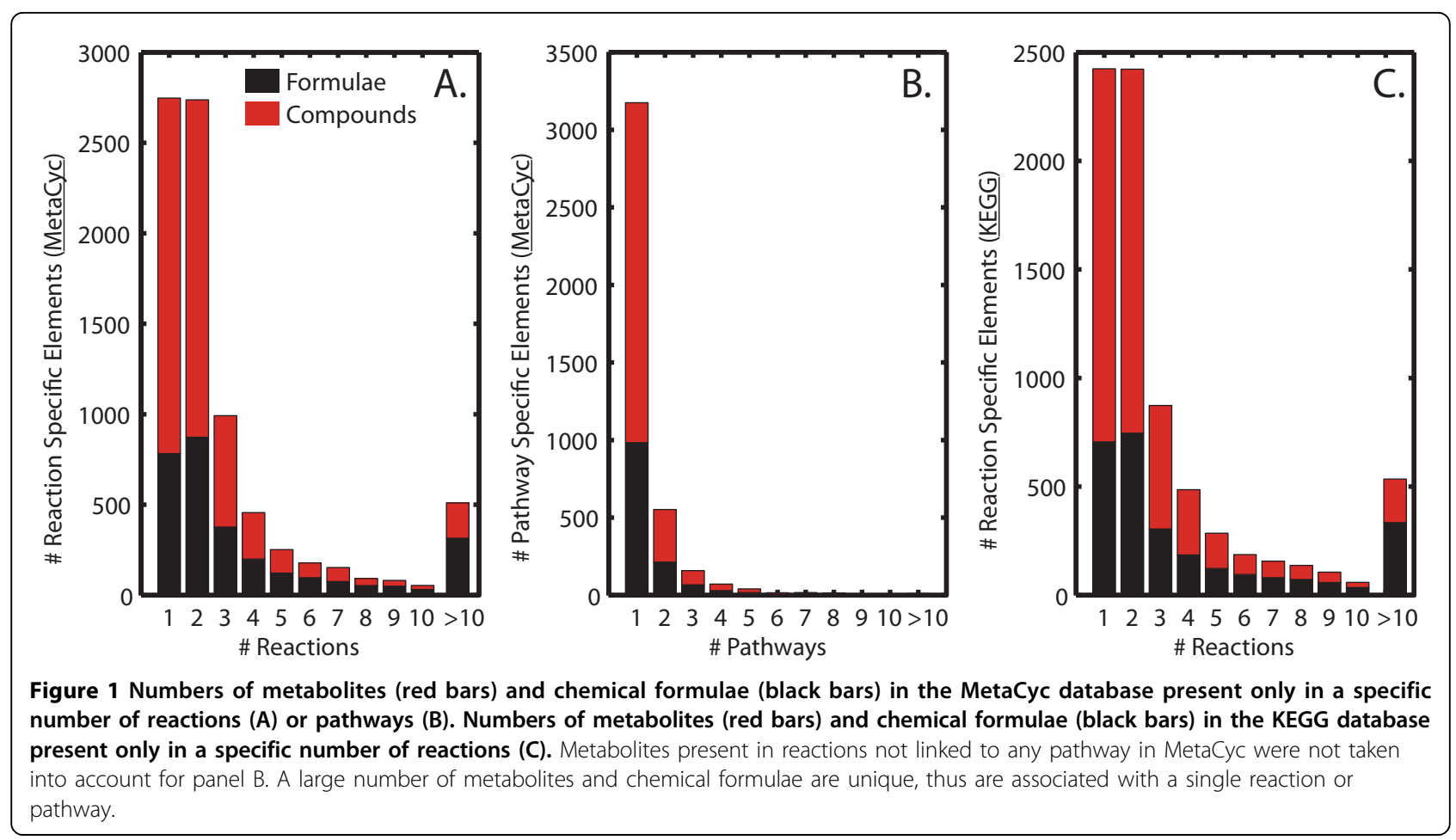

identified chemical formulae on to genome-scale metabolic network reconstructions or databases of metabolism offers an attractive, first step approach for the evaluation of the quality of genome annotation. The approach can also highlight gaps in the annotation when unexpected metabolites are identified [16-19].

\section{Chemical degeneracy around a local mass-value}

Shown in Figure 2 are two approaches for reducing chemical degeneracy around an observed value in chemical space. High mass accuracy mass spectrometers achieve an uncertainty of approximately $5 \mathrm{ppm}$. Because of this uncertainty, there can often be a range of chemical formulae that could correspond to an "inexact mass". To demonstrate, the HR2 chemical formula generator was used to find points in chemical space that are within $5 \mathrm{ppm}$ of folate $(441.1397 \mathrm{Da})$ (Figure 2A) [12]. Due to the large number of possible chemical formulae associated with this mass value, biological inference is impractical. However, the ratio of the monoisotopic peak intensity (M0) to the peaks with 1 (M1) or 2 (M2) more neutrons can be used to prioritize the likelihood that points in chemical space correspond to the measured mass (Figure 2C). This widely used approach is described in the Seven Golden Rules. Alternatively, knowledge of the number of carbon atoms and nitrogen atoms can be used to reduce the number of chemical formulae in the search space (Figure 2B).

\section{Chemical formula generation as the first step in restricting chemical space}

In Figure 3A, HR2 (with restrictions on allowable element ratios) was used to estimate the degeneracy in mass at $5 \mathrm{ppm}$ for a library of unique mass values. In addition, Figure 3B shows the increase in degeneracy for an unfiltered (brute force) formula generator. The restrictions reduce the degeneracy, but some compounds are lost when formula generators are restricted by heuristics such as Lewis senior rules and ring/double bond equivalents, and allowable element ratios. In both cases, the degeneracy becomes unmanageable as the mass of a compound increases.

Accurate mass alone is insufficient to identify the chemical formula for high mass metabolites. The degree to which degeneracy increases with mass was evaluated for 248,928 compouds, each having a unique mass. These masses were selected from the PubChem database by including only chemical compounds comprised of less than 201, 201, 7, 21, 7, and 7 (respectively) atoms of the elements $\mathrm{C}, \mathrm{H}, \mathrm{N}, \mathrm{O}, \mathrm{S}$, and $\mathrm{P}$ and having a mass of less than $1244 \mathrm{Da}$. The formulae of all these compounds could be generated by brute force. HR2, by design, uses heuristic filters to reduce the chemical formula search space; and therefore, it did not generate 11,380 of these formulae (Figure 3C). Most of these are unlikely to be biologically important (e.g. buckyballs: $\mathrm{C}_{60}$, tetrazete: $\mathrm{N}_{4}$ ). However, others, including ATP, taurine, and malate are of biological importance. These compounds 


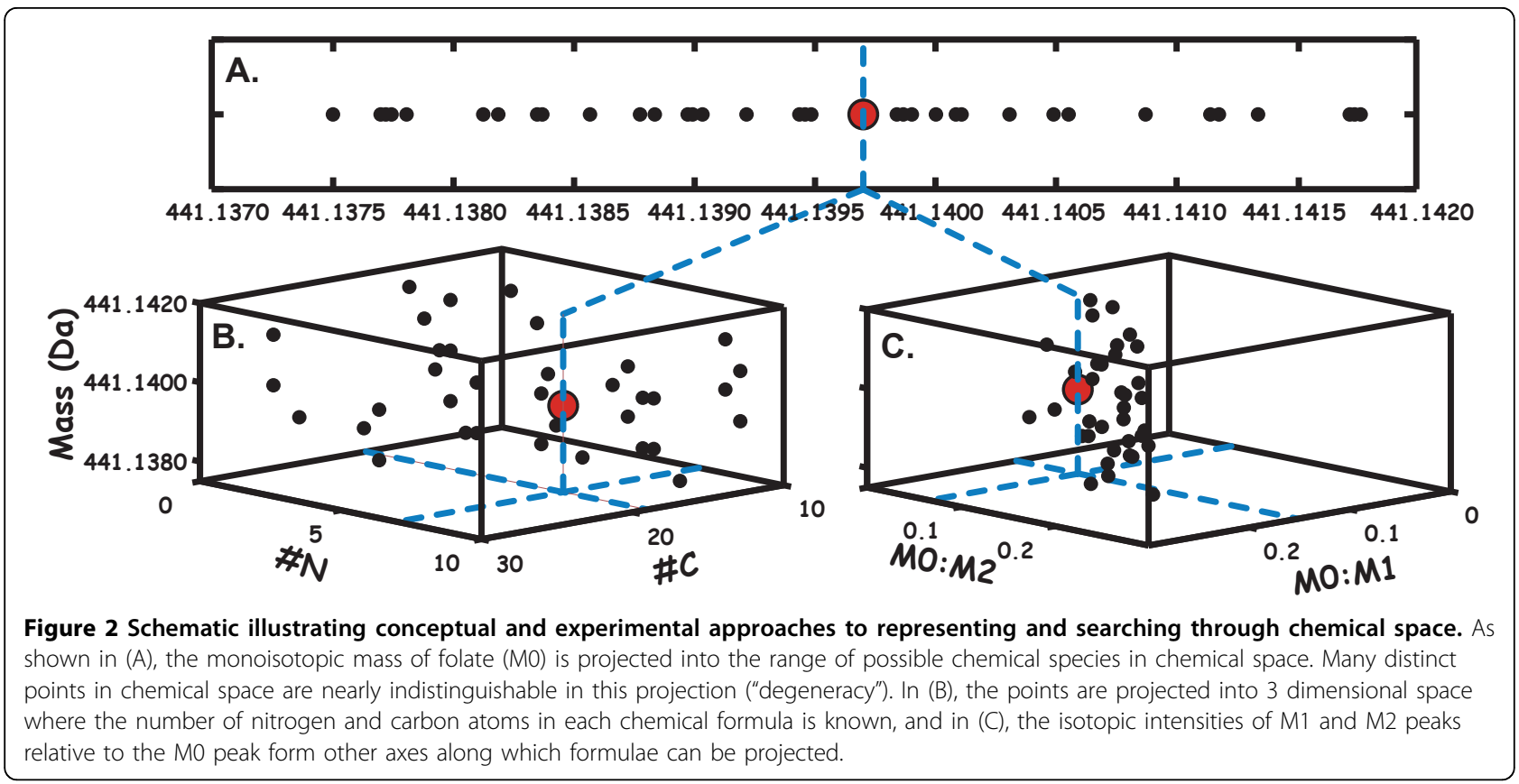

are excluded by the compiled version of HR2 by restricting the oxygen to carbon ratio. This variable can be easily changed in the source code of HR2 to a more liberal value as described in the Seven Golden Rules [12]. We conclude that some metabolomics experiments can benefit from a less restricted formula generator, though use of an unrestricted chemical formula generator greatly increases the search space around a mass value. It is important to note that in cases where a compound has constitutional or stereoisomers and therefore lacks an unique chemical formula, the formula can provide valuable information to narrow the search, often to

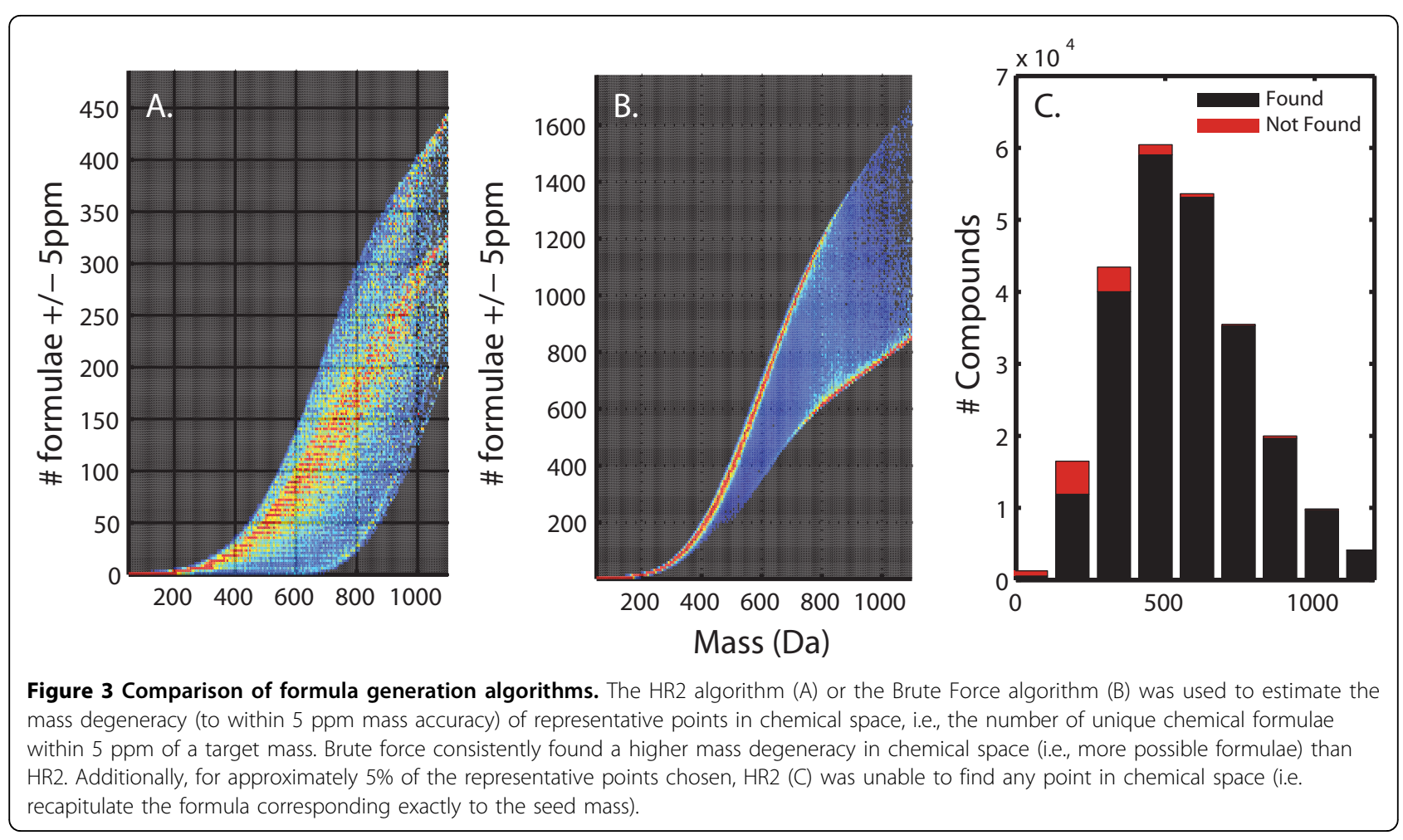


a given class of compounds (e.g. hexose), providing biological considerations.

\section{Defined C \& $N$ atom count for identification of a unique chemical formula}

By determining two dimensions in chemical space (the $\mathrm{C}$ and $\mathrm{N}$ count) the degeneracy of possible formulae is reduced. For the 248,928 chemically representative unique masses, we determined the degree to which specifying the $\mathrm{C}$ and $\mathrm{N}$ count determines a unique chemical formula (Figure 4). Using only knowledge from the HR2 chemical formula generator, unique mass defines chemical space location only for compounds less than approximately $500 \mathrm{Da}$ (Fig 4A), and only for $5 \%$ of all compounds. When the unconstrained chemical formula generator (brute force) was applied to the same masses, very few could be localized to a specific coordinate in chemical space ( $0.5 \%$ of all compounds). However in Figure 4 B \& C, determining only the number of carbon and nitrogen atoms for each mass reduces the degeneracy to a practical level (55\% have a unique formula with HR2 and 50\% with brute force).

\section{Comparison to relative isotopic peak intensities}

The third of the Seven Golden Rules requires that the relative intensities of the peaks with one (M1) and two (M2) more neutrons compared to the monoisotopic (M0) peak are within a specified deviation compared to the pattern predicted for a chemical formula according to natural isotopic abundance. To determine the degree to which specifying the $\mathrm{C}$ and $\mathrm{N}$ count reduces degeneracy in comparison to this rule, 10,000 masses were selected at random from the library of 248,929 unique masses. For each selected mass value, formulae were generated within $5 \mathrm{ppm}$; and for each, the relative intensity of the M1 and M2 isotopic peaks as compared to the monoisotopic peak (M0) was calculated. This was done using both HR2 (Fig $5 \mathrm{C} \& \mathrm{D}$ ) and brute force (Fig $5 \mathrm{~A} \& \mathrm{~B}$ ). Furthermore, to test whether SIL knowledge was able to reduce the degeneracy better than the ratios of isotopic peak intensities, the number of possible chemical formulae remaining when the $\mathrm{N}$ and $\mathrm{C}$ count are known was also determined. This analysis was carried out as a function of the uncertainty in relative isotopic peak intensity. At zero-uncertainty, the ratio of the isotopic peaks uniquely defines a location in chemical space. However, realistically, there will be uncertainty associated with intensity $[11,13]$. At $10 \%$ uncertainty in the intensity ratios, the distribution of masses that were better localized by SIL or relative isotopic peak intensities is shown (Fig 5 B \& D). Beyond approximately $500 \mathrm{Da}$, natural isotopic abundance information fails to achieve the improvement in chemical localization that SIL is capable of (typically a unique formula).

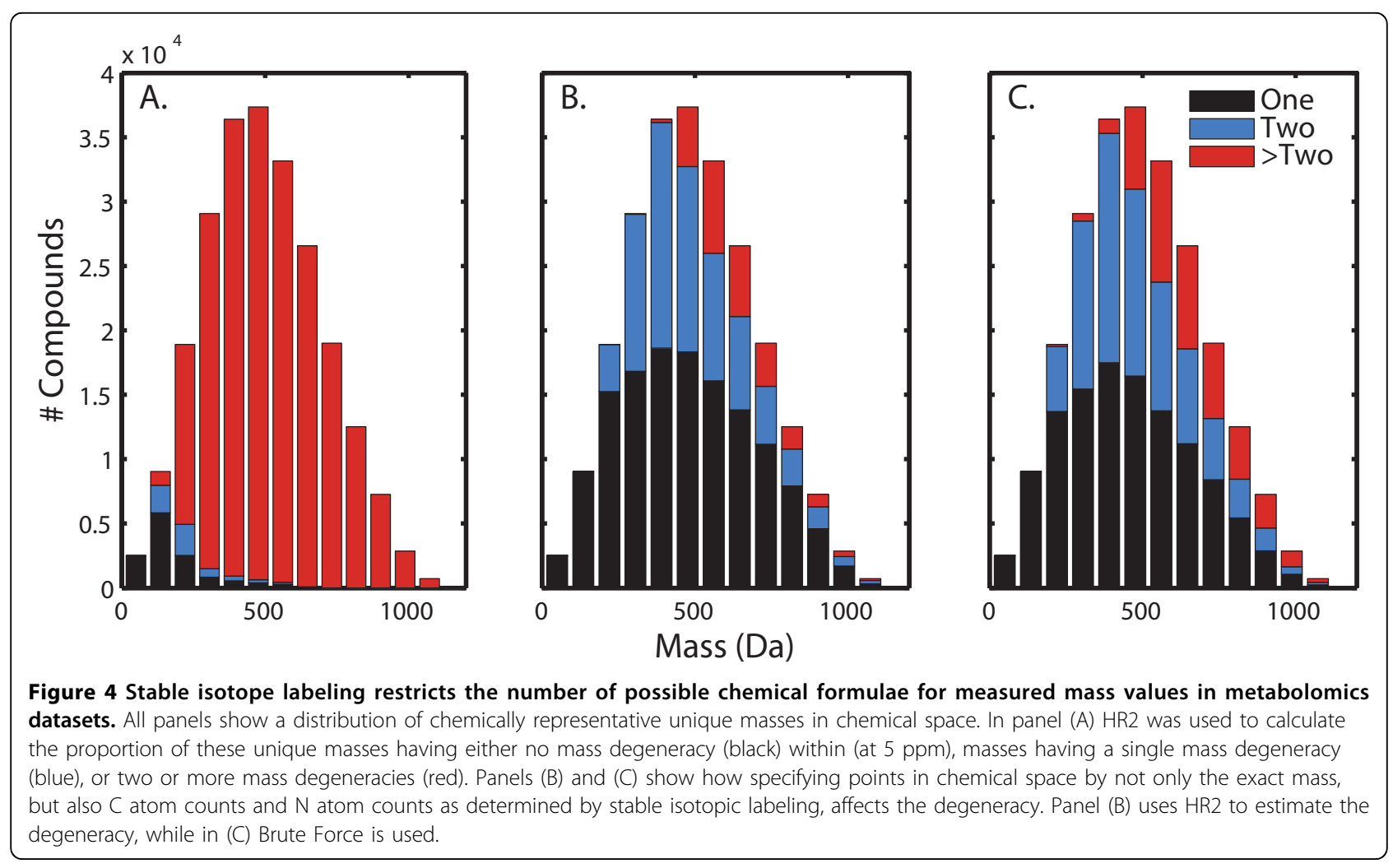




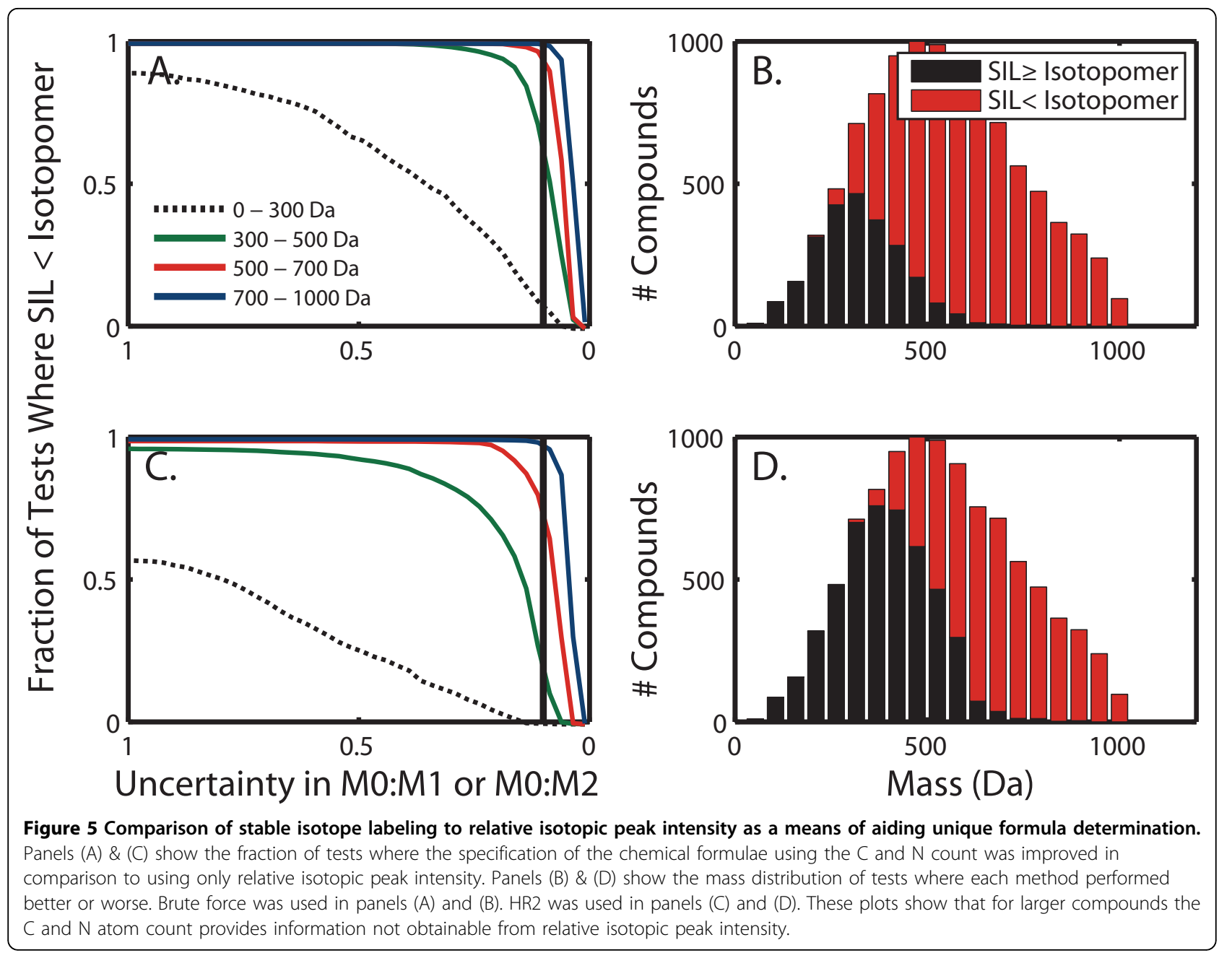

\section{Conclusions}

While mass spectrometry alone often cannot determine which isomer of a metabolite is present, our analysis has shown that pathway-specific metabolites and metabolites with unique chemical formulae exist. Thus, if the entire spectrum of chemical formulae for an organism's metabolites could be identified, clear designation of some metabolic pathways can be made.

To facilitate interpretation of metabolomics data, methods for identifying the chemical formula of detected features are greatly needed. A key deterrent to the identification of chemical formulae has historically stemmed from degeneracy, which increases with mass. We demonstrate here that the SIL method is better than existing methods at identification of chemical formulae for metabolites larger than $500 \mathrm{Da}$. This is achieved through determination of the $\mathrm{C}$ and $\mathrm{N}$ atom count. An additional advantage of the SIL method is that it functions well even when the relative error of the isotopic peak intensities is $>10 \%$, however, this method has the disadvantage that it requires additional experimentation. We have shown that the use of heuristic filters in chemical formula generation, while effective at reducing degeneracy and do not require additional experiments, runs the risk of ignoring biologically relevant metabolites. This study demonstrates that the SIL method reduces degeneracy enough that unfiltered chemical formula generation is feasible.

\section{Methods}

All figures and analyses were performed in Matlab 7.10.0 (R2010a) or Mathematica (v7.0.1).

\section{Identification of unique metabolites}

MetaCyc version 14.1 was downloaded on 8/4/2010 [20]. The following files were used: compounds.dat, reactions. dat, and pathways.dat. From this, pathways which are not "Super-Pathways" were selected. All reactions and their corresponding metabolites containing only elements $(C$, $\mathrm{H}, \mathrm{N}, \mathrm{O}, \mathrm{S}$, and $\mathrm{P}$ ) related to a pathway were identified. In total 8,741 metabolites were considered. When restricted by elements 7,782 remained. OF these, there were 4,178 
unique formulae. Each metabolite in each pathway was examined to determine if the same metabolite or a complementary chemical formula was described in any other pathway or reaction not linked to a pathway.

\section{Generation of chemically representative unique masses}

The PubChem database was downloaded on, October $6^{\text {th }}$, 2009[21]. Entries were imported with Mass $\geq 50$ and $\leq$ 2000 , not having non-natural isotopomers, and not having a charge explicitly stated in the molecular formula field (34,753,108 compounds). This list was then filtered to compounds that only have the following elements $(\mathrm{C}, \mathrm{H}$, $\mathrm{N}, \mathrm{O}, \mathrm{S}$, and $\mathrm{P}$ ), as these define the majority of biological metabolites (20,706,238 compounds). Further filtering to require $(\mathrm{C}, \mathrm{H}, \mathrm{N}, \mathrm{O}, \mathrm{S}$, and $\mathrm{P})$ to span the range of ([1:200], $[1: 200],[0: 6],[0: 20],[0: 6]$, and $[0: 6])$ respectively reduced the database size by $6.4 \%$. Of the remaining $19,378,002$ compounds, 248,928 have unique formulae. These chemically representative unique masses were used to perform the analysis presented here. Of the unique formulae in PubChem, 143,499 have a molecular weight greater than $500 \mathrm{Da}$. Although this ratio of heavy to light molecules is different than what would be found in MetaCyc (there are 1,833 out of 8,869 in MetaCyc that are between 500 and $2000 \mathrm{Da}$ ), the purpose in using PubChem is to attempt to explore a large chemical formula space.

\section{Comparison of formulae to those in the KEGG database}

From ftp:kegg/compounds, a custom script was written to parse this file and return only those compounds that are not charged and have a defined chemical formula (not a polymer and not having a generic R-group) [22]. Out of 11,221 molecules, there are 6,181 unique chemical formulae. Of the unique chemical formulae, 5,042 are comprised of only CHNOPS and 5014 are within the 50 to $2000 \mathrm{Da}$ mass range. There are 1,489 with a molecular weight greater than $500 \mathrm{Da}$.

\section{Determining the number of formulae within $5 \mathrm{ppm}$ by HR2}

The command line chemical formula generator was called for each of the unique masses described above [12]. The following string was issued to the program in order to constrain the possible formulae by the same constraints used for selecting the masses: "HR2-all-res. exe -C "test" -m MASS -t TOL -C 1-200 - H 1-200 -N 0-6 -O 0-20 -P 0-6 -S 0-6" where MASS is the neutral mass and TOL is the $5 \mathrm{ppm}$ window size. The text output by HR2 was parsed using a custom script to return chemical formulae (additional file 2).

Determining the number of formulae by brute force A custom script was written in Matlab to generate all possible combinations over the range $(\mathrm{C}, \mathrm{H}, \mathrm{N}, \mathrm{O}, \mathrm{S}, \mathrm{P})$ of ([1:200],
[1:200], [0:6], [0:20], [0:6], [0:6]) respectively. Formulae and corresponding masses within $5 \mathrm{ppm}$ were returned.

\section{Isotopic pattern generator}

A custom script was written in Matlab to generate relative isotopic peak intensities for a given chemical formula. The script uses multinomial probability distributions to calculate the exact abundance of the elemental isotopologues, one element at a time. The probabilities of a given isotomer for each element are binned on a user-defined mass-axis, and these vectors are then convolved to give the molecular isotopomer distribution patternthat includes all relevant elements.

\section{Author's contributions}

$\mathrm{BB}, \mathrm{RB}$, and CF developed the algorithms presented here. All authors contributed to experimental design and draft of the manuscript. All authors read and approved the final manuscript.

\section{Additional material}

Additional file 1: Table showing unique formulas and their pathways.

Additional file 2: Table showing KEGG formulas not defined by HR2.

List of abbreviations used

SIL: Stable Isotope Labeling; PPM: parts per million; TOF: Time of Flight; HR2: A chemical formula generator constrained by heuristics [12].

\section{Acknowledgements}

This work was part of the US Department of Energy Genomics Sciences program: ENIGMA is a Scientific Focus Area Program supported by the US Department of Energy, Office of Science, Office of Biological and Environmental Research, Genomics: GTL Foundational Science through contract DE-AC02-05CH11231 between Lawrence Berkeley National Laboratory and the U.S. Department of Energy and contract DE-SC0004665 to the University of California, Berkeley.

This article has been published as part of BMC Genomics Volume 12 Supplement 1, 2011: Validation methods for functional genome annotation. The full contents of the supplement are available online at http://www. biomedcentral.com/1471-2164/12?issue=S1.

\section{Author details}

'Department of GTL Bioenergy and Structural Biology, Life Sciences Division, Lawrence Berkeley National Laboratory, 1 Cyclotron Road, Berkeley, CA 94720, USA. ²Department of Earth and Planetary Science, Policy, and Management, University of California at Berkeley, Berkeley CA 94720, USA. ${ }^{3}$ Department of Environmental Science, Policy, and Management, University of California at Berkeley, Berkeley CA 94720, USA.

\section{Competing interests}

The authors declare that they have no competing interests.

\section{Published: 15 June 2011}

\section{References}

1. Yizhak K, Benyamini T, Liebermeister W, Ruppin E, Shlomi T: Integrating quantitative proteomics and metabolomics with a genome-scale metabolic network model. Bioinformatics 2010, 26(12):i255-260. 
2. Joyce AR, Palsson BO: The model organism as a system: integrating 'omics' data sets. Nat Rev Mol Cell Biol 2006, 7(3):198-210.

3. May P, Wienkoop S, Kempa S, Usadel B, Christian N, Rupprecht J, Weiss J, Recuenco-Munoz L, Ebenhoh O, Weckwerth W, et al: Metabolomics- and proteomics-assisted genome annotation and analysis of the draft metabolic network of Chlamydomonas reinhardtii. Genetics 2008, 179(1):157-166.

4. Baran R, Reindl W, Northen T: Mass spectrometry based metabolomics and enzymatic assays for functional genomics. Current Opinion in Microbiology 2009, 12(5):547-552.

5. Saghatelian A, Trauger S, Want E, Hawkins E, Siuzdak G, Cravatt B: Assignment of endogenous substrates to enzymes by global metabolite profiling. Biochemistry 2004, 43(45):14332-14339.

6. Oh YK, Palsson BO, Park SM, Schilling CH, Mahadevan R: Genome-scale reconstruction of metabolic network in Bacillus subtilis based on highthroughput phenotyping and gene essentiality data. J Biol Chem 2007, 282(39):28791-28799

7. Saito N, Robert M, Kitamura S, Baran R, Soga T, Mori H, Nishioka T, Tomita M: Metabolomics approach for enzyme discovery. Journal of Proteome Research 2006, 5(8):1979-1987.

8. Blow N: Metabolomics: Biochemistry's new look. Nature 2008, 455(7213):697-700.

9. De Vos RCH, Moco S, Lommen A, Keurentjes JJB, Bino RJ, Hall RD: Untargeted large-scale plant metabolomics using liquid chromatography coupled to mass spectrometry. Nat Protoc 2007, 2(4):778-791.

10. Böcker S, Rasche F: Towards de novo identification of metabolites by analyzing tandem mass spectra. Bioinformatics 2008, 24(16):i49-i55,

11. Kind T, Fiehn O: Metabolomic database annotations via query of elemental compositions: Mass accuracy is insufficient even at less than 1 ppm. BMC Bioinformatics 2006, 7:234.

12. Kind T, Fiehn O: Seven golden rules for heuristic filtering of molecular formulas obtained by accurate mass spectrometry. BMC Bioinformatics 2007, 8(1):105.

13. Ipsen A, Want EJ, Ebbels TM: Construction of confidence regions for isotopic abundance patterns in LC/MS data sets for rigorous determination of molecular formulas. Anal Chem 2010, 82(17):7319-7328.

14. Nakamura Y, Kanaya S, Sakurai N, lijima Y, Aoki K, Okazaki K, Suzuki H, Kitayama M, Shibata D: A tool for high-throughput prediction of molecular formulas and identification of isotopic peaks from large-scale mass spectrometry data. Plant Biotechnol 2008, 25(4):377-380.

15. Böcker S, Letzel MC, Lipták Z, Pervukhin A: SIRIUS: decomposing isotope patterns for metabolite identification. Bioinformatics 2009, 25(2):218-224

16. Giavalisco P, Hummel J, Lisec J, Inostroza AC, Catchpole G, Willmitzer L: High-Resolution Direct Infusion-Based Mass Spectrometry in Combination with Whole C-13 Metabolome Isotope Labeling Allows Unambiguous Assignment of Chemical Sum Formulas. Analytical Chemistry 2008, 80(24):9417-9425.

17. Giavalisco P, Kohl K, Hummel J, Seiwert B, Willmitzer L: C-13 IsotopeLabeled Metabolomes Allowing for Improved Compound Annotation and Relative Quantification in Liquid Chromatography-Mass Spectrometry-based Metabolomic Research. Analytical Chemistry 2009, 81(15):6546-6551.

18. Hegeman AD, Schulte CF, Cui Q, Lewis IA, Huttlin EL, Eghbalnia H, Harms AC, Ulrich EL, Markley JL, Sussman MR: Stable isotope assisted assignment of elemental compositions for metabolomics. Analytical Chemistry 2007, 79(18):6912-6921.

19. Baran R, Bowen BP, Bouskill NJ, Brodie EL, Yannone SM, Northen TR Metabolite Identification in Synechococcus sp. PCC 7002 Using Untargeted Stable Isotope Assisted Metabolite Profiling. Analytical Chemistry 2010, 82(21):9034-9042.

20. Caspi R, Foerster H, Fulcher C, Kaipa P, Krummenacker M, Latendresse M, Paley S, Rhee S, Shearer A, Tissier C: The MetaCyc Database of metabolic pathways and enzymes and the BioCyc collection of Pathway/Genome Databases. Nucleic Acids Research 2008, 36(Database issue):D623.

21. Wang Y, Xiao J, Suzek TO, Zhang J, Wang J, Bryant SH: PubChem: a public information system for analyzing bioactivities of small molecules. Nucleic Acids Res 2009, 37(Web Server issue):W623-633.

22. Kanehisa M, Goto S: KEGG: kyoto encyclopedia of genes and genomes. Nucleic Acids Res 2000, 28(1):27-30.
doi:10.1186/1471-2164-12-S1-S6

Cite this article as: Bowen et al:: Improved genome annotation through untargeted detection of pathway-specific metabolites. BMC Genomics 2011 12(Suppl 1):S6.

\section{Submit your next manuscript to BioMed Central and take full advantage of:}

- Convenient online submission

- Thorough peer review

- No space constraints or color figure charges

- Immediate publication on acceptance

- Inclusion in PubMed, CAS, Scopus and Google Scholar

- Research which is freely available for redistribution

Submit your manuscript at www.biomedcentral.com/submit
Ciomed Central 